\title{
Um roteiro para avaliação ontológica de modelos de sistemas de informação
}

\author{
Viviane Nogueira Pinto de Oliveira
}

\author{
Mestre em Ciência da Informação
}

Mauricio Barcellos Almeida

Professor Adjunto da Escola de Ciência da Informação da UFMG

No âmbito dos sistemas de informação, uma ontologia pode ser usada tanto como um componente do sistema quanto como uma referência, ou seja, um padrão de comparação para avaliar a representatividade do modelo subjacente ao sistema. $O$ presente artigo explora 0 segundo tipo de aplicação, discutindo o uso ontologias na criação de modelos conceituais. Apresentam-se fundamentos do processo de criação de modelos para sistemas de informação e exemplos de ontologias utilizadas como referência. Propõe-se um roteiro para avaliação ontológica de modelos sob o ponto de vista da linguagem de modelagem e descreve-se um estudo de caso para testar a viabilidade da proposta. Espera-se contribuir para a pesquisa que envolve ontologias e modelagem conceitual, através de resultados empíricos.

Palavras-chave: Ontologias; Representação do conhecimento; Sistemas de informação; Modelos conceituais.

\section{A list of topics for ontological evaluation of information system models}

Within the scope of automated information systems an ontology can be used as a component of a system or as a reference. Such reference is a type of standard comparison employed to evaluate the ability of the model underlying the system to represent the world. The present article explores the latter application, discussing the usefulness of ontologies in conceptual modeling processes. We present modeling essentials and examples 
of ontologies used as references. Then, we propose a list of topics to perform an ontological evaluation of models from the point of view of modeling language, and describe a case study to test the feasibility of the proposal. We hope to contribute to the research that relates ontologies and conceptual modeling through empirical results.

Keywords: Ontologies; Knowledge representation; Information systems; Conceptual models.

Recebido em 18.11.2009 Aceito em 10.01.2011

\section{Introdução}

Originado na Filosofia, o termo ontologia passou a ser utilizado em Representação do Conhecimento ainda na década de 60, para referenciar um artefato de software ou linguagem formal utilizada em sistemas declarativos. Nos anos 80, surgiram pesquisas na Engenharia de Software para aplicação de ontologias em sistemas de informação (SI) procedurais. Essa nova abordagem buscava entender processos, que têm lugar ao longo da atividade de modelagem conceitual.

A modelagem conceitual é uma fase do desenvolvimento de SI, que tem por finalidade descrever a realidade, representando os processos de interesse para desenvolvimento de um sistema. Um problema comum de SI em uso nas instituições é a dificuldade de interoperabilidade entre sistemas desenvolvidos por diferentes equipes, em diferentes períodos e para propósitos diversos. Tal problema tem origem principalmente em atividades de modelagem deficientes, conduzidas de forma ad-hoc e sem correspondência com o mundo real (SMITH; WELTY, 2001; GUARINO, 1998).

Uma alternativa para buscar a interoperabilidade entre sistemas é comparar a linguagem utilizada na criação do modelo a um padrão, uma referência, uma ontologia bem fundamentada. Ontologias bem fundamentadas são ontologias filosóficas e suas variações, bem como ontologias de alto nível. Criadas a partir de princípios filosóficos, essas ontologias auxiliam na solução de problemas de integração entre sistemas, ao fornecer subsídios para avaliar modelos conceituais, facilitando a comunicação humana, característica da atividade de modelagem (WAND; STOREY; WEBER, 1999).

Para adotar tal abordagem, é relevante conhecer as ontologias utilizadas em modelagem conceitual, suas variações já testadas e os princípios ontológicos subjacentes. Ainda que se trate de abordagem de fins dos anos 1980 (WAND; WEBER, 1989), ainda têm sido amplamente utilizada e os resultados considerados satisfatórios (GUARINO; GUIZZARDI, 2006). Inserindo-se nesse contexto, o presente artigo contextualiza a atividade de modelar, descreve as principais ontologias utilizadas para fins de modelagem de SI e apresenta um roteiro baseado 
em ontologias para avaliação de modelos conceituais. Além disso, de forma a testar a viabilidade do roteiro, descreve-se um estudo de caso com modelos de sistemas reais de instituições públicas brasileiras. Esperase contribuir para a pesquisa que relaciona ontologias à modelagem, consolidando o roteiro proposto através de investigação empírica.

$O$ restante do presente artigo está organizado conforme segue. A Seção 2 introduz os modelos e apresenta um breve histórico, desde os primeiros modelos de dados para SI. A Seção 3 contextualiza a e apresenta as ontologias utilizadas como referência para a criação de modelos. A seção 4 propõe o roteiro e apresenta o estudo de caso. A Seção 5 apresenta conclusões e perspectivas futuras.

\section{Modelos e Modelagem}

A atividade de modelar tem início por meio de processos sensoriais e intelectuais, quando a mente de uma pessoa decompõe o fenômeno e seleciona recortes da realidade a representar. No âmbito da atividade humana, modelos são criados para diversos fins como, por exemplo, representação, simulação, previsão, dentre outros. Nesse contexto, destaca-se o desenvolvimento de SI. O restante dessa seção apresenta características básicas dos modelos e da atividade de modelar, bem como um breve histórico sobre uso de modelos de dados para SI.

\subsection{Características e funções dos modelos}

Modelos são instrumentos de comunicação em que a informação é apreendida e transferida através de uma estrutura de representação, utilizados em inúmeras atividades humanas para representar a realidade de forma simplificada. Os modelos possuem características que dependem do objeto a ser representado e dos objetivos de quem modela. De fato, podem existir diferentes modelos para representar uma mesma parte da realidade, o que não significa que um ou outro esteja incorreto.

Os modelos e a atividade de modelar são objeto de estudo de diversos campos científicos e diferentes pontos de vista. Segundo Kühne (2005), a atividade de modelar envolve: i) mapeamento, que resulta em aproximações seletivas; ii) redução, pois um modelo não mapeia todos os atributos originais; e iii) pragmatismo, uma vez que modelos atuam como substitutos de seus originais. Sayão (2001) apresenta como principais funções dos modelos: i) função aquisitiva, através da qual a informação de interesse é coletada e ordenada; ii) função lógica, a qual explica como ocorre um fenômeno; iii) função normativa, a qual permite comparar fenômenos a outros similares; iv) função sistemática, através da qual a realidade é descrita como um conjunto de sistemas interligados; e v) função construtiva, através da qual teorias e leis são construídas. Sánchez, Cavero e Marcos (2005) classificam os modelos de acordo com a sua natureza em: i) modelos abstratos, nos quais as representações são descritas a partir de regras de linguagem (por ex., modelos matemáticos); e ii) modelos concretos, nos quais as representação são criadas a partir de 
instâncias que possuem os requisitos necessários para gerar ideias ou conceitos (por ex., uma maquete).

A despeito de seu tipo ou função, os modelos cumprem importante papel na prática científica e na produção de conhecimento. Permitem que um cientista confronte a realidade experimental com a realidade do mundo e verifique hipóteses por meio de mecanismos de abstração e de simulação. No contexto de ciência, Frigg (2006) destaca três tipos de questões sobre modelos: questões de natureza ontológica, de natureza epistemológica e de natureza semântica.

As questões de natureza ontológica dizem respeito a definir o que são modelos dentre: objetos físicos, objetos ficcionais, estruturas da teoria dos conjuntos, descrições, equações, ou combinações dos anteriores. Nas questões de natureza epistemológica, os modelos são considerados meios de aprendizado e, a partir deles, é possível descobrir características do sistema representado. As questões de natureza semântica dizem respeito à função de representação dos modelos.

No contexto semântico, cabe distinguir os modelos de fenômenos, as teorias e os modelos de dados. Um modelo de fenômeno lida com problemas de representação científica. Tal modelo é uma estrutura que torna verdadeiras as sentenças de uma teoria. Por sua vez, um modelo de dados é uma visão idealizada de dados obtidos por observação direta. Modelos de dados possuem um papel fundamental na confirmação de teorias porque são claros e de fácil interpretação.

\subsection{Modelos de SI}

Modelos de dados proliferam nas organizações, como forma de representar entidades sociais, físicas e tecnológicas. A atividade de criar modelos de dados constitui-se em uma importante estratégia para a comunicação e a cooperação em organizações. Para Fox e Gruninger (1998), um modelo de dados provê uma linguagem para definição dos processos organizacionais, a qual origina uma infraestrutura de comunicação. Em última instância, o principal objetivo de um modelo de dados é obter uma visão sobre o funcionamento de uma instituição.

$\mathrm{Na}$ atividade de criar modelos de SI, representam-se os principais processos de um contexto específico objetivando a construção do sistema. A etapa em que são criados modelos com vistas ao entendimento humano é conhecida como modelagem conceitual. Os modelos conceituais são obtidos a partir de abstrações de aspectos da realidade, da perspectiva de uma pessoa ou grupo de pessoas. As abstrações são formas de especificar as entidades e as relações entre as entidades, em um domínio do conhecimento de interesse para o sistema em desenvolvimento.

A modelagem conceitual de SI, como conhecida hoje, é resultado de pesquisa produzida nos últimos 50 anos. As primeiras iniciativas para especificação de modelos de dados datam de final dos anos 1950 (YOUNG; KENT, 1958; BOSAK et al., 1962). Em tais iniciativas, a criação de modelos privilegiava as necessidades ditadas por estruturas de dados computacionais. Nos anos 1960, a pesquisa em bancos de dados deu 
origem aos modelos conhecidos como modelos lógicos. Esses modelos não fazem referência direta a aspectos físicos (codificação), mas ainda têm limitações, se utilizados em modelagem conceitual. Nos anos 1970, surgiram os modelos semânticos utilizados em modelagem conceitual (ABRIAL, 1974; JARDINE, 1976; CHEN, 1976). A principal característica dos modelos semânticos é a facilidade de entendimento. Nos anos 90, as propostas de modelagem orientadas a objeto se tornaram populares. A Unified Modeling Language (UML) buscava uniformizar as notações da orientação a objeto, reunindo melhores práticas de outras iniciativas (BOOCH, 1993; RUMBAUGH et al., 1991; JACOBSON et al., 1992).

De fato, ao longo dos anos, a pesquisa sobre modelos conceituais tem sido motivada pela busca de formas cada vez mais apuradas de representar a realidade em SI. Segundo Mylopoulos (1992, p. 3), a modelagem conceitual é "[...] a atividade de formalmente descrever aspectos do mundo físico e social ao nosso redor para propósitos de entendimento e comunicação" ${ }^{1}$. Entretanto, os modelos semânticos dispõem de um conjunto limitado de constructos para a tarefa de modelagem conceitual. O modelo $E R$, por exemplo, pressupõe que a realidade pode ser articulada por dois constructos apenas (a saber, entidade e relacionamento).

Como alternativa, Guarino (1998) destaca a necessidade de uma conceitualização comum entre SI. Segundo o autor, a interoperabilidade entre sistemas é possível apenas se as linguagens subjacentes aos modelos possuem conceitualizações que se sobrepõem em algum nível. Em última instância, apenas uma conceitualização compartilhada, produto da comunicação humana, pode proporcionar melhorias na modelagem. Ontologias são alternativas para especificar tal conceitualização.

\section{Ontologias aplicadas à modelagem de SI}

Em um ambiente caracterizado pela facilidade em estabelecer redes, as limitações de representação dos modelos conceituais resultam em dificuldades de integração entre sistemas. Essa dificuldade é muitas vezes resultado da modelagem inconsistente adotada nos primórdios da computação (SMITH; WELTY, 2001). Uma alternativa para esse reduzir esse tipo de problema são os modelos baseados em ontologias.

O restante da presente seção contextualiza a pesquisa em ontologias no âmbito da modelagem de SI, apresenta as ontologias utilizadas como referência, descreve exemplos de ontologias filosóficas, suas variações e ontologias de alto-nível.

\subsection{Ontologias de SI}

Ontologias têm sido estudadas na Inteligência Artificial desde os anos 1970. Nos anos 1990, a Web Semântica fez crescer a demanda por ontologias. O estudo das ontologias tem se caracterizado pela coexistência

${ }^{1}[\ldots]$ the activity of formally describing some aspects of the physical and social world around us for purposes of understanding and communication. 
de abordagens interdisciplinares. Guarino e Giaretta (1995), por exemplo, citam sete interpretações possíveis para o termo ontologia. Ao longo dos anos, diversos autores têm estudado o tema (GENESERETH; NILSSON, 1987; GRUBER, 1993; VICKERY, 1997; SØERGUEL, 1997; GUARINO, 1998; SOWA, 2000; SMITH, 2003). Uma fundamentação teórica detalhada sobre ontologias está além do escopo do presente artigo. Ainda assim, considerações relevantes para os propósitos do artigo são apresentadas no restante dessa seção.

Segundo Smith (1998), do ponto de vista filosófico, só pode existir uma ontologia. Para lidar com a pluralidade de usos do termo, distinguem-se dois tipos de ontologias: a ontologia real, com a qual se estuda como o universo é organizado; e a ontologia epistemológica, relacionada à tarefa de conceitualizar um domínio. A ontologia epistemológica corresponde a um artefato, ou seja, a ontologia como é interpretada na Engenharia de Software e na Representação do Conhecimento.

Para Guarino (1998), uma ontologia descreve o significado dos símbolos utilizados em SI de acordo com uma visão particular de mundo. As ontologias são classificadas em duas dimensões principais, de acordo com o impacto que produzem nos SI: a dimensão temporal corresponde ao uso da ontologia em SI, em tempo de desenvolvimento ou em tempo de execução; a dimensão estrutural diz respeito ao uso da ontologia, como um componente de um banco de dados.

Fonseca (2007) distingue ontologias de SI e ontologias para SI. No primeiro caso, a ontologia é utilizada em modelagem e exemplos são os trabalhos de Green e Roseman (2005), Fettke e Loss (2005), Holten, Dreiling e Becker (2004), dentre outros. No segundo caso, a ontologia é componente do SI e descreve o vocabulário de um domínio. Exemplos de ontologias para SI são as pesquisas de Crubézy e Munsen (2004), de Sycara e Paolucci (2004), de Oberle et al., (2004), dentre outras.

Através de ontologias de SI, conforme a definição de Fonseca (2007), é possível avaliar a capacidade de representação de linguagens de modelagem. Comparam-se os constructos da linguagem aos constructos ontológicos de uma ontologia de referência. Considera-se, no escopo desse trabalho, que ontologias de referência ${ }^{2}$ são: i) ontologias filosóficas, as quais buscam interpretar o mundo; ii) variações de ontologias filosóficas, as quais correspondem a adaptações com fins específicos; e iii) ontologias de alto nível, as quais contêm representações genéricas, passíveis de utilização em domínios diversos. Exemplos de ontologias filosóficas são a ontologia de Bunge e a ontologia de Chisholm. Um exemplo de variação de ontologia filosófica muito utilizado em SI é a Bunge-Wand-Weber (BWW). Exemplos de ontologias de alto nível são: a Descriptive Ontology for Linguisitics and Cognitive Engineering (DOLCE) (MASOLO et al., 2003), a General Formal Ontology (GFO) (HERRE et al.,

\footnotetext{
2 Existem variações do termo com significado similar: ontologias de base, ontologias fundamentais,
} dentre outras. 
2006) e a Unified Foundational Ontology (UFO) (GUIZZARDI; WAGNER, 2004).

A seção 3.2 descreve brevemente as principais ontologias de referência utilizadas em modelagem de SI.

\subsection{Ontologias de referência para modelagem conceitual}

Chisholm ${ }^{3}$ desenvolveu uma ontologia filosófica realista de senso comum (CHISHOLM, 1996). O realismo corresponde à presunção de que a realidade do mundo existe independente da cognição humana (SMITH, 2003). Milton e Kazmierczak (2004) descrevem a semântica das categorias da ontologia de Chisholm, distinguindo as entidades do mundo entre conceitos estáticos (indivíduos, atributos, classes, relações) e conceitos dinâmicos (relações temporais, estados).

Os estudos de Bunge ${ }^{4}$ resultaram em uma ontologia filosófica realista, a qual considera a possibilidade do conhecimento humano objetivo, desde que este se baseie no método científico (BUNGE, 1977). $\mathrm{Na}$ ontologia de Bunge, coisa e propriedade são os constructos atômicos usados para descrever o mundo: o mundo é feito de coisas, as quais têm propriedades; propriedades não podem ter propriedades; duas coisas interagem quando a estória de uma delas, manifestada como uma sequência de estados, poderia ter sido diferente se a outra não existisse.

A pesquisa pioneira sobre ontologias de SI é resultado de uma extensão da teoria de Bunge conhecida como BWW (WAND; WEBER, 1990; WAND; STOREY; WEBER, 1999). Trata-se de uma proposta que fornece bases teóricas para avaliar práticas de modelagem e a capacidade de representação das linguagens. Wand e Weber (1990) descrevem critérios, denominados completeza ontológica e transparência ontológica, para verificar possibilidades de mapeamento entre os construtos da linguagem de modelagem e os constructos ontológicos definidos pela BWW.

O projeto WonderWeb Foundational Ontologies Library tem por objetivo desenvolver linguagens para representação de ontologias. A primeira etapa do projeto especificou uma ontologia baseada em lógica Modal. Essa ontologia, denominada DOLCE (MASOLO et al., 2003), tem por objetivo descrever categorias ontológicas subjacentes ao senso comum humano.

A GFO (HERRE et al., 2006) é uma ontologia de alto nível utilizada no âmbito da Biomedicina. Além dessa aplicação principal, a GFO é utilizada como fundamento ontológico para modelagem conceitual. A ontologia consiste de três níveis denominados nível abstrato, nível núcleo e nível básico, os quais incluem objetos, processos, além de módulos especializados.

A UFO (GUIZZARDI; WAGNER, 2004) é resultado da combinação da GFO, da DOLCE e de princípios da metodologia OntoClean (GUARINO;

\footnotetext{
3 Roderick Chisholm, filósofo norte-americano (1916 - 1999).

${ }^{4}$ Mario Bunge, filósofo e físico argentino (1919-?).
} 
WELTY, 2002). Como síntese de outras ontologias, a UFO oferece maior número de constructos para avaliação de linguagens de modelagem conceitual. Consiste de três camadas: UFO-A, que define o núcleo ontológico; UFO-B, relacionada a processos; e UFO-C, relacionada às esferas sociais e aspectos linguísticos.

Apesar das diversas abordagens, não existe uma ontologia de referência genericamente aceita para uso em modelagem. Ainda assim, existem diversas iniciativas de pesquisa que fazem uso de ontologias de referência, o que indica a viabilidade da proposta. Exemplos são os trabalhos de Evermann (2003), de Shanks, Tansley e Weber (2003), de Holten, Dreiling e Becker (2004), de Milton e Kazmierczak (2000).

\section{0 roteiro proposto: avaliação ontológica de SI}

A presente seção descreve o roteiro proposto, o qual contempla as etapas para avaliação de modelos de SI a partir de ontologias de referência (Seção 4.1). Além disso, estuda-se a viabilidade da proposta, através de estudo de caso em diagramas de SI reais (Seção 4.2). Os diagramas foram elaborados na linguagem UML e os seus constructos comparados a uma ontologia de referência.

\subsection{As etapas e os instrumentos}

O roteiro proposto organiza os procedimentos em quatro etapas principais. De forma a facilitar o entendimento, o QUADRO 1 apresenta de forma resumida as etapas e os instrumentos:

QUADRO 1 Quadro resumo das etapas do roteiro

\begin{tabular}{c|l|l}
\hline Etapa & \multicolumn{1}{|c|}{ Tarefa } & \multicolumn{1}{c}{ Instrumentos utilizados } \\
\hline Etapa 1 & Seleção dos diagramas & $\begin{array}{l}\text { Especificações de requisitos; informações sobre o } \\
\text { contexto de produção dos sistemas. }\end{array}$ \\
\hline Etapa 2 & $\begin{array}{l}\text { Sistematização de critérios de avaliação; } \\
\text { estabelecimento de condições para aplicação }\end{array}$ & $\begin{array}{l}\text { Diagramas de classe selecionados; ontologia } \\
\text { BWW. }\end{array}$ \\
\hline Etapa 3 & Mapeamento dos diagramas para a ontologia & $\begin{array}{l}\text { Especificação UML, diagramas de classe, critérios } \\
\text { de avaliação. }\end{array}$ \\
\hline Etapa 4 & Proposição de novos diagramas & $\begin{array}{l}\text { Mapeamento obtido na etapa três e ferramenta } \\
\text { CASE. }\end{array}$ \\
\hline
\end{tabular}

A primeira etapa consiste na seleção de diagramas. Como é necessário um mínimo de uniformidade na escolha de diagramas a avaliar, adotaram-se critérios que dizem respeito: à equipe de desenvolvimento, ao período do desenvolvimento e aos futuros usuários.

A segunda etapa envolve duas questões relevantes: a sistematização de critérios de avaliação e o estabelecimento de condições para sua aplicação. A sistematização dos critérios é importante na busca por princípios metodológicos generalizáveis. A relevância em determinar condições de aplicação dos critérios reside no fato de que nem todos os diagramas são passíveis de avaliação. Além disso, tanto a sistematização de critérios quanto as condições podem variar em função da escolha de diferentes ontologias de referência. 
O QUADRO 2 apresenta uma versão resumida do conjunto de critérios (C) para avaliação de diagramas $\mathrm{UML}^{5}$. Esses critérios são baseados na pesquisa sobre mapeamento entre a BWW e a UML, em particular na abordagem de Evermann (2005). Vários termos utilizados na descrição dos critérios tem origem na especificação da UML e na BWW.

\section{QUADRO 2 Critérios para avaliação dos diagramas}

\begin{tabular}{|c|c|}
\hline Critério & Descrição do critério (C) \\
\hline $\mathrm{C} 1$ & $\begin{array}{l}\text { Somente entidades substanciais no mundo são representadas como objetos. Uma coisa é } \\
\text { equivalente ao objeto na UML. Se uma entidade é substancial significa que ela é material, física. }\end{array}$ \\
\hline $\mathrm{C} 2$ & $\begin{array}{l}\text { Propriedades das coisas na ontologia devem ser representadas como atributos UML. O conceito de } \\
\text { propriedade na BWW equivale ao conceito de atributo na UML. }\end{array}$ \\
\hline $\mathrm{C} 3$ & $\begin{array}{l}\text { Conjuntos de propriedades mútuas devem ser representados como atributos de classes de } \\
\text { associação; as propriedades mútuas, ao contrário das intrínsecas, são compartilhadas entre coisas. }\end{array}$ \\
\hline C4 & $\begin{array}{l}\text { Uma classe de associação não deve possuir métodos ou operações. Ela não pode representar } \\
\text { entidades substanciais porque, neste caso, seus atributos seriam intrínsecos a uma coisa } \\
\text { substancial. }\end{array}$ \\
\hline C5 & $\begin{array}{l}\text { Ao alterar propriedades mútuas, os métodos e as operações que alteram os atributos da classe de } \\
\text { associação devem ser representados por classes que fazem parte da associação e não por classes de } \\
\text { associação. }\end{array}$ \\
\hline C6 & $\begin{array}{l}\text { Uma classe de associação deve possuir um atributo. Se uma classe de associação representa } \\
\text { propriedades mútuas, então deve possuir pelo menos um atributo. }\end{array}$ \\
\hline $\mathrm{C7}$ & $\begin{array}{l}\text { Uma classe de associação não deve estar associada à outra classe; propriedades na ontologia não } \\
\text { podem possuir propriedades mútuas relacionadas a outras propriedades ou coisas. }\end{array}$ \\
\hline $\mathrm{C} 8$ & $\begin{array}{l}\text { Uma classe de associação não deve participar em relacionamentos de generalização. Classes de } \\
\text { associação são propriedades na ontologia e não podem ser generalizadas ou especializadas. }\end{array}$ \\
\hline C9 & $\begin{array}{l}\text { Uma classe de associação representa um conjunto de propriedades mútuas originadas em uma } \\
\text { interação. Se uma propriedade se originou em interações diferentes, deve ser representada em } \\
\text { outra classe de associação. }\end{array}$ \\
\hline $\mathrm{C} 10$ & $\begin{array}{l}\text { Classes de objetos que exibem comportamentos ou atributos ou classes de associação adicionais em } \\
\text { relação a outros objetos da mesma classe, devem ser representados como subclasses } \\
\text { especializadas. }\end{array}$ \\
\hline $\mathrm{C} 11$ & $\begin{array}{l}\text { Todo objeto agregado UML deve consistir de pelo menos duas partes. Na ontologia, se uma coisa } \\
\text { composta é formada por apenas uma parte, então ela é a própria parte. }\end{array}$ \\
\hline $\mathrm{C} 12$ & $\begin{array}{l}\text { Todo objeto agregado UML deve possuir pelo menos um atributo ou participar em uma associação. } \\
\text { Na ontologia, uma coisa composta possui uma propriedade emergente, caso contrário não é coisa } \\
\text { composta. }\end{array}$ \\
\hline C13 & $\begin{array}{l}\text { Uma classe UML possui um atributo ou participa em uma associação. Se coisas possuem } \\
\text { propriedades, então as classes que representam conjuntos de objetos devem possuir atributos ou } \\
\text { participar de associação. }\end{array}$ \\
\hline $\mathrm{C} 14$ & $\begin{array}{l}\text { A identificação (ID) dos objetos não deve ser representada como atributo. Na ontologia, coisas são } \\
\text { identificadas pelos conjuntos de valores das propriedades e não existe um equivalente para o } \\
\text { identificador. }\end{array}$ \\
\hline $\mathrm{C} 15$ & $\begin{array}{l}\text { O conjunto de valores de atributos (propriedades mútuas e intrínsecas) deve identificar unicamente } \\
\text { um objeto; o conjunto de valores das propriedades de uma coisa deve ser capaz de representá-la } \\
\text { como única. }\end{array}$ \\
\hline $\mathrm{C} 16$ & $\begin{array}{l}\text { Uma classe especializada deve definir maior número de atributos, operações, ou participar em maior } \\
\text { número de associações do que a classe geral. }\end{array}$ \\
\hline $\mathrm{C} 17$ & $\begin{array}{l}\text { Toda associação ordinária deve ser uma classe de associação. A associação ordinária é distinta da } \\
\text { associação de composição ou de agregação. }\end{array}$ \\
\hline $\mathrm{C} 18$ & $\begin{array}{l}\text { Todo objeto deve ter ao menos uma operação. Na ontologia, todas as coisas podem mudar e as } \\
\text { operações representam mudanças. }\end{array}$ \\
\hline
\end{tabular}

O estabelecimento de condições para aplicação dos critérios de avaliação tem por objetivo uniformizar o processo e evitar incoerências. $\mathrm{Na}$ verdade, essas condições restringem o uso de alguns critérios em relação a certos constructos dos diagramas. Alguns critérios só são aplicáveis a classes de associação, outros apenas a classes de objetos. Por

\footnotetext{
${ }^{5}$ Pressupõem-se conhecimento básico de termos da UML.
} 
exemplo, o critério C11 pode ser aplicado apenas em entidades que mantém relação parte-todo.

O QUADRO 3 descreve as condições adotadas para a aplicação de critérios a diagramas. Alguns tipos de constructos comuns observados nos diagramas são classes, atributos, classes de associação, associações e multiplicidade. Ainda no QUADRO 3, "V" significa que a condição é verdadeira, " $F$ " que a condição é falsa e "NA" que a condição não se aplica.

\section{QUADRO 3 Conjunto de condições adotadas para a aplicação dos critérios}

\begin{tabular}{|c|c|c|c|}
\hline $\boldsymbol{C}$ & Aplicar? & $\begin{array}{c}\text { Constructo } \\
\text { analisado }\end{array}$ & Condição a verificar \\
\hline$C 1$ & Sim & $\begin{array}{l}\text { Classe }^{6} \\
\text { ou } \\
\text { Classe de } \\
\text { associação }\end{array}$ & $\begin{array}{l}\text { - Se o constructo analisado é uma classe e corresponde a uma coisa na } \\
\text { ontologia, o constructo atende ao critério (V); } \\
\text { - Se o constructo analisado é classe de associação UML e corresponde a } \\
\text { uma coisa na ontologia, o constructo não atende ao critério (F); }\end{array}$ \\
\hline$C 2$ & Sim & $\begin{array}{l}\text { Classe } \\
\text { ou } \\
\text { Classe de } \\
\text { associação }\end{array}$ & $\begin{array}{l}\text { - Se o constructo analisado é uma classe de associação e corresponde a } \\
\text { uma propriedade na ontologia, o constructo atende ao critério (V); } \\
\text { - Se o constructo analisado é uma classe e corresponde a uma } \\
\text { propriedade na ontologia, o constructo não atende ao critério (F); } \\
\text { - Se o constructo analisado não corresponde a uma propriedade na } \\
\text { ontologia, o critério não é aplicável (NA). }\end{array}$ \\
\hline C3 & Sim & $\begin{array}{l}\text { Classe } \\
\text { ou } \\
\text { Classe de } \\
\text { associação }\end{array}$ & $\begin{array}{l}\text { - Se o constructo analisado é uma classe de associação e corresponde a } \\
\text { uma propriedade mútua na ontologia, o constructo atende ao critério } \\
\text { (V); } \\
\text { - Se o constructo analisado é uma classe e corresponde a uma } \\
\text { propriedade mútua na ontologia, o constructo não atende ao critério } \\
\text { (F); } \\
\text { - Se o constructo analisado não corresponde a uma propriedade na } \\
\text { ontologia, o critério não é aplicável (NA). }\end{array}$ \\
\hline C4 & Não & $\begin{array}{l}\text { Classe de } \\
\text { associação }\end{array}$ & $\begin{array}{l}\text { - Este critério não foi utilizado, pois nenhuma operação foi representada } \\
\text { nos diagramas selecionados; }\end{array}$ \\
\hline C5 & Não & $\begin{array}{l}\text { Classe de } \\
\text { associação }\end{array}$ & $\begin{array}{l}\text { - Este critério não foi utilizado, pois nenhuma operação foi representada } \\
\text { nos diagramas selecionados; }\end{array}$ \\
\hline C6 & Sim & $\begin{array}{l}\text { Classe de } \\
\text { associação }\end{array}$ & $\begin{array}{l}\text { - Se o constructo é uma classe de associação e possui atributos, o } \\
\text { critério é atendido }(V) \text {; } \\
\text { - Se o constructo é uma classe de associação e não possui atributos, o } \\
\text { critério não é atendido (F); }\end{array}$ \\
\hline$C 7$ & Sim & $\begin{array}{l}\text { Classe de } \\
\text { associação }\end{array}$ & $\begin{array}{l}\text { - Se o constructo é uma classe de associação e não está associado à } \\
\text { outra classe, o critério é atendido }(V) \text {; } \\
\text { - Se o constructo é uma classe de associação e está associado à outra } \\
\text { classe, o critério não é atendido (F); }\end{array}$ \\
\hline C8 & Sim & $\begin{array}{c}\text { Classe de } \\
\text { associação }\end{array}$ & $\begin{array}{l}\text { - Se o constructo é uma classe de associação e possui relacionamentos } \\
\text { de generalização, o critério não é atendido (F); } \\
\text { - Se o constructo é uma classe de associação e não possui } \\
\text { relacionamentos de generalização, o critério é atendido (V); }\end{array}$ \\
\hline C9 & Sim & $\begin{array}{l}\text { Classe de } \\
\text { associação }\end{array}$ & $\begin{array}{l}\text { - Se todas as propriedades são originadas na interação que corresponde } \\
\text { à classe de associação, o critério é atendido }(V) \text {; } \\
\text { - Se há propriedades da classe de associação originadas em outra } \\
\text { interação que dá origem a outra classe de associação, o critério não é } \\
\text { atendido }(F) \text {; }\end{array}$ \\
\hline
\end{tabular}

\footnotetext{
${ }^{6} \mathrm{Na}$ aplicação dos critérios, classes são consideradas conjuntos de objetos que mantém a mesma descrição. Daqui em diante, utiliza-se apenas a denominação "classe".
} 


\begin{tabular}{|c|c|c|c|}
\hline C10 & Sim & Classe & $\begin{array}{l}\text { - Se o constructo analisado refere-se a uma coisa na ontologia e faz } \\
\text { parte de uma hierarquia, o critério é atendido }(V) \text {; } \\
\text { - Se o constructo analisado refere-se a uma coisa na ontologia, mas não } \\
\text { faz parte de uma hierarquia, o critério não é atendido }(F) \text {; }\end{array}$ \\
\hline$C 11$ & Sim & Classe & $\begin{array}{l}\text { - Se o constructo analisado faz parte de uma relação parte-todo e a } \\
\text { relação tem pelo menos duas partes, o critério é atendido (V); } \\
\text { - Se o constructo analisado faz parte de uma relação parte-todo, mas a } \\
\text { relação tem apenas uma parte, o critério não é atendido (F); } \\
\text { - Se o constructo analisado não faz parte de uma relação parte-todo, o } \\
\text { critério não é aplicável (NA); }\end{array}$ \\
\hline C12 & Sim & Classe & $\begin{array}{l}\text { - Se o constructo analisado faz parte de uma relação parte-todo e possui } \\
\text { pelo menos um atributo, o critério é atendido }(V) \text {; } \\
\text { - Se o constructo analisado faz parte de uma relação parte-todo, mas } \\
\text { não possui atributo, o critério não é atendido }(F) \text {; } \\
\text { - Se o constructo analisado não faz parte de uma relação parte-todo, o } \\
\text { critério não é aplicável }(N A) \text {; }\end{array}$ \\
\hline C13 & Sim & Classe & $\begin{array}{l}\text { - Se o constructo analisado participa de uma associação ou possui pelo } \\
\text { menos um atributo, o critério é atendido (V); } \\
\text { - Se o constructo analisado não participa de uma associação e não possui } \\
\text { atributo, o critério não é atendido ( } F) \text {; }\end{array}$ \\
\hline C14 & Sim & Classe & $\begin{array}{l}\text { - Se o constructo possui atributos e nenhum deles é atributo identificador } \\
\text { (ID), o critério é atendido (V); } \\
\text { - Se o constructo possui atributos e pelo menos um deles é atributo } \\
\text { identificador, o critério não é atendido (F); } \\
\text { - Se o constructo não possui atributos, o critério não se aplica (NA); }\end{array}$ \\
\hline C15 & Sim & Classe & $\begin{array}{l}\text { - Se o constructo possui atributos que são capazes de identificá-lo } \\
\text { univocamente, o critério é atendido (V); } \\
\text { - Se o constructo possui atributos que não são capazes de identificá-lo } \\
\text { univocamente, o critério não é atendido (F); } \\
\text { - Se existir um atributo identificador (ID) ele será desconsiderado do } \\
\text { conjunto de atributos da classe/objeto (NA); }\end{array}$ \\
\hline C16 & Sim & Classe & $\begin{array}{l}\text { - Se o constructo corresponde a uma coisa na ontologia, então ele é } \\
\text { especializado e possui maior número de atributos ou associações que o } \\
\text { constructo genérico, o critério é atendido }(V) \text {; } \\
\text { - Se o constructo corresponde a uma coisa na ontologia, é especializado } \\
\text { e não possui número maior de atributos ou associações que o } \\
\text { constructo genérico, o critério não é atendido }(F) \text {; }\end{array}$ \\
\hline$C 17$ & Não & Associação & $\begin{array}{l}\text { - Este critério não foi utilizado, pois não se mostrou relevante no âmbito } \\
\text { dos diagramas selecionados; }\end{array}$ \\
\hline C18 & Não & Classe & $\begin{array}{l}\text { - Este critério não foi utilizado, pois nenhuma operação foi representada } \\
\text { nos diagramas selecionados; }\end{array}$ \\
\hline
\end{tabular}

Cabe destacar que a determinação de condições sobre a aplicabilidade dos critérios não é trivial. Por exemplo, para verificar se uma entidade UML corresponde a uma coisa na ontologia é preciso tomar por base a definição da entidade na especificação de requisitos. A especificação, entretanto, nem sempre continha uma definição capaz de subsidiar a interpretação sobre a natureza da entidade. Uma avaliação precisa sobre a substancialidade dos objetos UML está além do escopo do presente trabalho e será considerada em trabalhos futuros.

$\mathrm{Na}$ terceira etapa, através dos critérios de avaliação, mapeiam-se constructos UML dos diagramas para os constructos da ontologia. A especificação UML é consultada para apreensão da semântica dos constructos. O mapeamento consiste em uma sequência de tarefas: 
especificação do constructo analisado, comparação entre o construto UML e o constructo ontológico, especificação do critério aplicado e proposição de alterações. A quarta etapa consiste em propor mudanças nos diagramas originais. Novos diagramas são gerados por uma ferramenta CASE, alimentada com os resultados obtidos no mapeamento. Exemplos do mapeamento da etapa 3 e de diagramas modificados na etapa 4 são apresentados a seguir na seção 4.2.

\subsection{Aplicação do roteiro: estudo de caso}

No estudo de caso foram utilizados modelos de SI implementados e em uso na administração pública do Estado de MG. O principal objetivo do estudo de caso foi avaliar a viabilidade prática do roteiro proposto, aplicando-o a diagramas utilizados em sistemas reais. No restante da presente seção, apresentam-se o estudo de caso e os resultados da aplicação do roteiro, bem como considerações registradas ao longo da pesquisa.

\subsubsection{Diagramas selecionados}

Selecionou-se um grupo de especificações, dentre as disponíveis, de forma a obter um mínimo de uniformidade considerando-se: i) equipe responsável: as especificações foram produzidas pela mesma equipe de desenvolvimento; ii) período: todas as especificações foram desenvolvidas quase que simultaneamente, entre o final de 2005 e o início de 2008; iii) cliente: todas as especificações foram contratadas pelo mesmo órgão público; e iv) usuários: os futuros usuários do sistema não eram sempre os mesmos, mas mantinham similaridades (cargos, nível educacional, conhecimento dos processos).

A partir dessas considerações, decidiu-se sobre quais especificações de requisitos forneceriam diagramas para avaliação. As especificações dos seguintes sistemas foram escolhidas: i) Portal para Compras; ii) Sistema de Controle de Telefonia; e iii) Sistema de Gestão de Documentos. Finalmente, foram relacionadas, dentre o grupo de especificações, 120 diagramas para avaliação através dos critérios ontológicos.

Dentre as experiências obtidas ao longo da pesquisa, cabe destacar a discussão com a equipe acerca do nível de abstração dos diagramas. Observou-se que alguns diagramas, ainda que citados como de modelagem conceitual, eram típicos do nível lógico (Sistema de Telefonia); outros, de fato, eram claramente diagramas de nível conceitual (Sistema de Gestão de Documentos). Após a discussão, percebeu-se que os limites entre esses níveis não são bem definidos, mesmo no âmbito de uma mesma equipe. Alguns diagramas seriam assim pouco indicados para a pesquisa em curso.

Do ponto de vista da aplicabilidade da ontologia à avaliação de diagramas, considerou-se a quantidade de entidades substanciais presentes. Entidades substanciais são denominadas coisas na BWW, uma ontologia que considera que mundo é feito de coisas físicas que possuem 
propriedades. Dessa forma, não foram analisados modelos compostos apenas interações e propriedades.

Em última instância, buscou-se diagramas representativos de modelagem conceitual. A partir das considerações obtidas com a equipe e aquelas relacionadas a aplicabilidade da ontologia, selecionou-se uma subamostra para avaliação, dentre cento e vinte diagramas disponíveis. Foram selecionados sete diagramas representativos dos casos normalmente encontrados no universo de diagramas considerado.

\subsubsection{A aplicação de critérios e o mapeamento entre diagrama e ontologia}

A FIG.1 apresenta como exemplo o diagrama denominado Módulo de Acompanhamento de Tramitação do Processo Legislativo, extraído do sistema Portal de Compras. Um fragmento dos resultados do mapeamento é apresentado na QUADRO 4, onde apresentam-se o constructo original (UML), o critério aplicado e o resultado da aplicação, o respectivo constructo BWW e as alterações propostas.

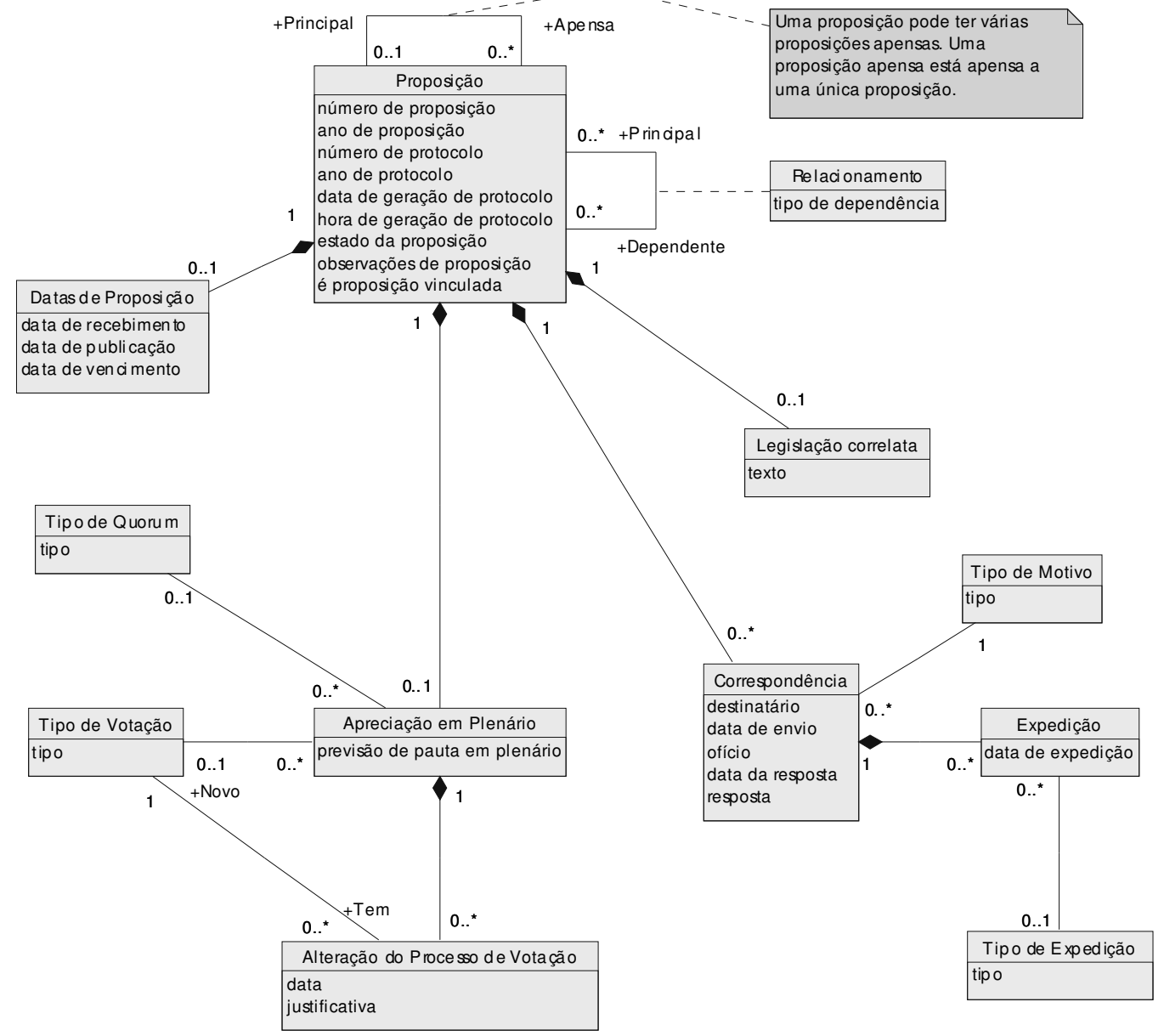

FIGURA 1 - Exemplo de diagrama avaliado 
QUADRO 4 Fragmento da tabela de mapeamento de diagrama apresentado na FIG. 4

\begin{tabular}{|c|c|c|c|c|c|c|c|}
\hline $\begin{array}{c}\text { Constructo } \\
\text { analisado }\end{array}$ & $\begin{array}{l}\text { Construct } \\
\text { o UML }\end{array}$ & & $\begin{array}{l}\text { irité } \\
\text { plica }\end{array}$ & & $\begin{array}{l}\text { Entidade } \\
\text { na BWW }\end{array}$ & $\begin{array}{l}\text { Alterações } \\
\text { Propostas }\end{array}$ & Observação \\
\hline \multirow{7}{*}{$\begin{array}{l}\text { Datas de } \\
\text { proposição }\end{array}$} & \multirow{7}{*}{ Classe } & \multirow{7}{*}{ C } & 01 & $F$ & \multirow{7}{*}{ Propriedade } & \multirow{7}{*}{$\begin{array}{l}\text { Passa a ser: } \\
\text { atributo de } \\
\text { Proposição. }\end{array}$} & \multirow{7}{*}{$\begin{array}{l}\text { Datas de proposição não é } \\
\text { representado, mas os seus } \\
\text { atributos são transferidos } \\
\text { para a classe Proposição. }\end{array}$} \\
\hline & & & 02 & $F$ & & & \\
\hline & & & 11 & $F$ & & & \\
\hline & & & 12 & $V$ & & & \\
\hline & & & 13 & $V$ & & & \\
\hline & & & 14 & $V$ & & & \\
\hline & & & 15 & $V$ & & & \\
\hline \multirow{7}{*}{$\begin{array}{l}\text { Apreciação } \\
\text { em Plenário }\end{array}$} & \multirow{7}{*}{ Classe } & \multirow{7}{*}{ C } & 01 & $F$ & \multirow{7}{*}{ Interação } & \multirow{7}{*}{$\begin{array}{l}\text { Passa a ser: } \\
\text { classe de } \\
\text { associação entre } \\
\text { Parlamentar e } \\
\text { Proposição }\end{array}$} & \multirow{7}{*}{$\begin{array}{l}\text { Não existe a classe } \\
\text { Parlamentar, a qual é } \\
\text { necessária para } \\
\text { representar a interação } \\
\text { que origina a classe de } \\
\text { associação Apreciação em } \\
\text { Plenário. }\end{array}$} \\
\hline & & & 03 & $F$ & & & \\
\hline & & & 11 & $F$ & & & \\
\hline & & & 12 & $V$ & & & \\
\hline & & & 13 & $V$ & & & \\
\hline & & & 14 & $V$ & & & \\
\hline & & & 15 & $V$ & & & \\
\hline \multirow{5}{*}{$\begin{array}{l}\text { Tipo de } \\
\text { votação }\end{array}$} & \multirow{5}{*}{ Classe } & \multirow{5}{*}{ C } & 01 & $F$ & \multirow{5}{*}{ Propriedade } & \multirow{5}{*}{$\begin{array}{l}\text { Passa a ser: } \\
\text { atributo da classe } \\
\text { de associação } \\
\text { Apreciação em } \\
\text { Plenário. }\end{array}$} & \multirow{5}{*}{$\begin{array}{l}\text { O atributo desta classe não } \\
\text { é representado, pois sendo } \\
\text { apenas um atributo e com } \\
\text { nome similar ao nome da } \\
\text { classe Tipo de votação que } \\
\text { já o representa. }\end{array}$} \\
\hline & & & 02 & $F$ & & & \\
\hline & & & 13 & V & & & \\
\hline & & & 14 & $V$ & & & \\
\hline & & & 15 & $V$ & & & \\
\hline
\end{tabular}

\subsubsection{Novos diagramas}

Uma vez realizado o mapeamento, foram propostos novos diagramas. As modificações são identificadas no QUADRO 4, no campo "alterações propostas", onde aparece a expressão "passa a ser". A FIG. 2 apresenta um exemplo de diagrama modificado após a aplicação dos critérios ao diagrama original da FIG. 1.

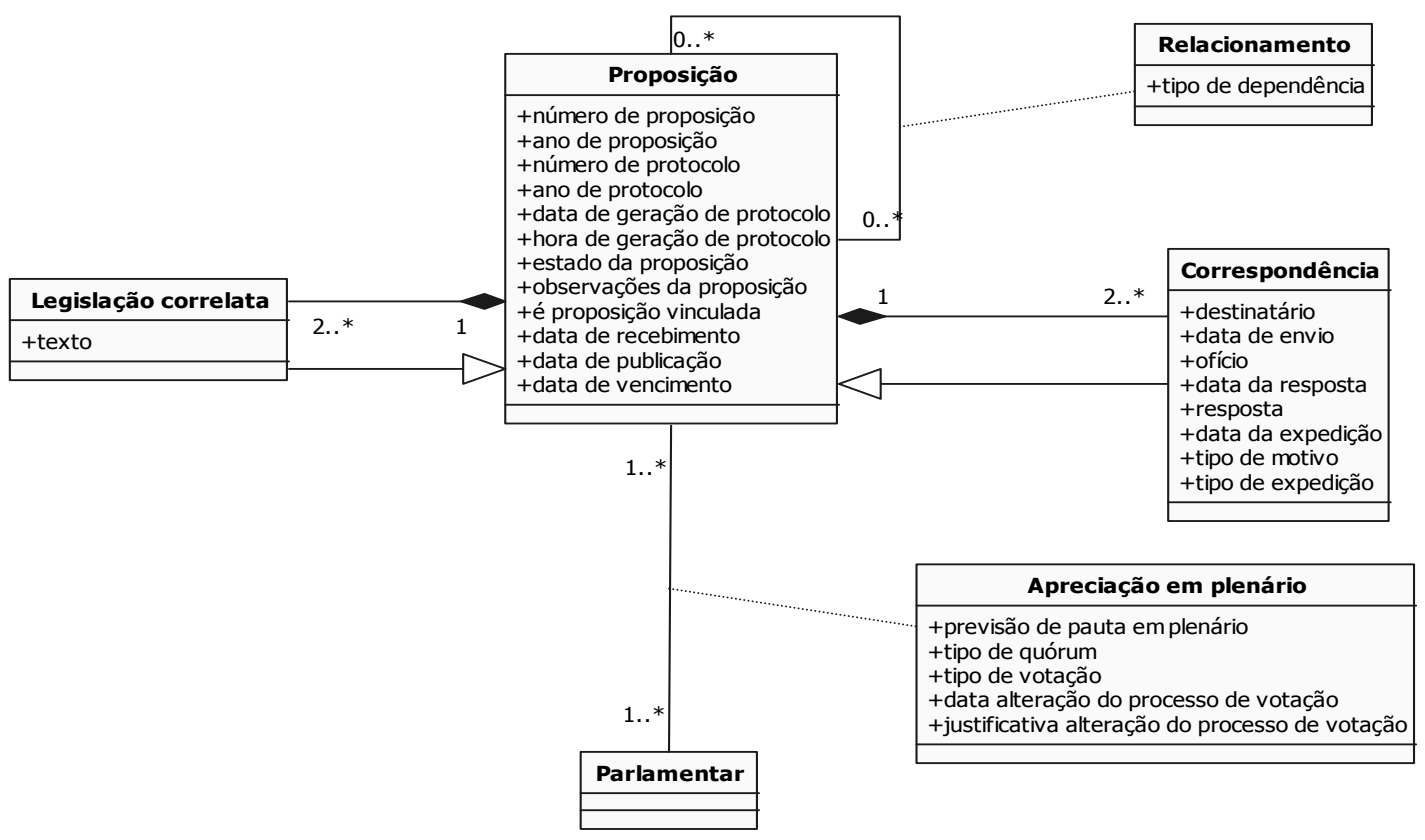

FIGURA 2 - Diagrama modificado

A análise do conjunto de diagramas possibilitou a percepção de padrões de modelagem que, em geral, não atendem a certos critérios 
estipulados pela ontologia. Os resultados, mesmo que qualitativos, contribuem para a percepção de que os processos de modelagem nem sempre são conduzidos por princípios ontologicamente fundamentados. Cabe ainda citar que apresentar aqui todos os diagramas, originais e os modificados, não é possível por limitações de espaço. Todos os diagramas e informações complementares sobre o contexto de sua produção estão disponíveis em Oliveira (2009).

\section{Considerações finais}

Esse artigo propôs um roteiro para a avaliação de diagramas de modelagem de SI com o uso de ontologias. Apresentaram-se fundamentos da aplicação de ontologias à modelagem de SI. Em seguida, apresentouse o roteiro e um estudo de caso, bem como observações registradas na pesquisa empírica. Cabe agora apresentar considerações finais sobre os resultados, além de propostas de trabalhos futuros.

O roteiro proposto baseou-se em critérios para a avaliação de diagramas UML, sob uma perspectiva ontológica. Os critérios se mostraram adequados para o estudo de caso, pois permitiram a aplicação direta de princípios de ontologias à UML. Além disso, foram estabelecidas condições que auxiliaram a definir restrições para a aplicação dos critérios. As condições permitiram o mapeamento entre diagrama e ontologia (FIG. 6) e, em seguida, foram propostos novos diagramas.

Observou-se que os diagramas não contêm apenas uma representação do contexto em que serão utilizados, mas também entidades inexistentes e concernentes a aspectos tecnológicos. De fato, parece ser prática comum entre analistas incluir na modelagem conceitual, aspectos importantes para a etapa subseqüente de implementação. Apesar da utilidade dessas entidades na codificação, elas geram inconsistências sob a ótica da ontologia. Neste sentido, o estudo de caso conduzido encontrou dificuldades para a seleção de amostras, já que grande parte dos diagramas continha situações desse tipo.

Diante das dificuldades encontradas para mapear certas entidades para a ontologia, discutiu-se com a equipe de desenvolvimento sobre que tipo de modelagem é normalmente adotado. A equipe de desenvolvimento informou que, de fato, os analistas agregam elementos da implementação ao modelo, em favor da praticidade na implementação. Em alguns casos ficou clara a predominância de modelos típicos da modelagem no nível lógico. A omissão da modelagem conceitual parece resultado de pressões comerciais, orçamento e prazos.

A despeito das dificuldades encontradas, muitos dos diagramas do estudo de caso se mostraram boas oportunidades de teste para o roteiro proposto, revelando comportamentos passíveis de análise pela ontologia. Diferenças significativas na forma de modelar foram verificadas, o que permitiu vislumbrar a ontologia como uma boa alternativa para estabelecer padrões de modelagem. A necessidade de estabelecer tais padrões se tornou mais evidente com a percepção de que as 
inconsistências encontradas são oriundas de práticas comuns na modelagem.

A proposta pioneira para avaliação de modelagem a partir de ontologias, conforme já mencionado, é de Wand e Weber. Wand e Weber (1989) relatam que a dificuldade em obter dados reais levou a coleta dados para testes empíricos em diagramas criados por alunos. Acredita-se que uma das contribuições da presente pesquisa reside na tentativa de validação do roteiro através de diagramas reais, que resultaram em SI implementados. Apesar da variedade de estudos que aplicam ontologias à modelagem de sistemas, pesquisas empíricas, como o estudo de caso apresentado, evidenciam detalhes que contribuirão para pesquisa futuras. Destaca-se, por exemplo, como contribuições: a necessidade de discussão prévia sobre o que a equipe de desenvolvimento considera ser modelagem conceitual; a necessidade de verificação da aplicabilidade dos critérios ao material empírico, a partir da definição de condições; dentre outros.

A pesquisa permitiu concluir sobre a necessidade de reflexões sobre as práticas de modelagem correntes. Esse foi o resultado prático mais significativo, uma vez que os sistemas objeto da pesquisa já estão implementados, e dessa forma não passíveis de algum tipo de correção. Tornou-se clara a necessidade de agregar esse tipo de avaliação crítica em metodologias de desenvolvimento dos sistemas, no âmbito considerado no estudo de caso. Procurou-se propor melhorias, fomentar a reflexão sobre casos reais, bem como recolher registros de uma investigação empírica para uso futuro.

Cabe ainda destacar o papel da ontologia, da forma como é entendida na Filosofia, como alternativa para melhor entendimento da realidade, resultando na possibilidade da criação de modelos de maior qualidade. Princípios ontológicos, fundamentados em teorias metafísicas, são uma boa oportunidade para rever formas e práticas para organizar a informação. Nesse contexto amplo, incluem-se as possibilidades de modelagem.

Vislumbram-se, como oportunidades para trabalhos futuros, dentre outras: o uso de uma amostra mais significativa de diagramas, que permita a evolução do roteiro para uma metodologia; ii) a avaliação da substancialidade das entidades UML presentes nos diagramas, e não apenas aquela baseada em informações das especificações de requisitos; e iii) a avaliação de diagramas representativos de processos e não apenas de classes.

\section{Agradecimentos}

-Ao prof. Robson Mateus do DCC-UFMG, pela cessão dos diagramas. -O co-autor é bolsista da CAPES - processo BEX 4071/09-3. 


\section{Referências}

ABRIAL, J. R. Data semantics. In: KLIMBIE, J. W.; KOFFEMAN, K. L. (Eds.). IFIP WORKING CONFERENCE DATA BASE MANAGEMENT, 1., 1974, Amsterdam. Proceedings... Amsterdam: North-Holland, 1974. p. 1-60.

$\mathrm{BOOCH}, \mathrm{G}$. Object-oriented analysis and design with applications. 2nd. Redwood: Cummings, 1993. 608 p.

BOSAK, R. et al. An information algebra: phase 1 report - language structure group of the CODASYL development committee. Communications of the ACM, New York, v. 5, n. 4, p. 190-204, 1962.

BUNGE, M. Ontology I: the furniture of the world. Dordrecht: Reidel, 1977. 376 p. (Treatise on basic Philosophy, v. 3).

CHEN, P. P. S. The entity-relationship model-toward a unified view of data. ACM Transactions on Database Systems, v. 1, n. 1, p. 9-36, 1976.

CHISHOLM, R. A Realistic theory of categories - an essay on ontology. 1 ed. Cambridge: Cambridge University Press, 1996. 146 p.

CRUBÉZY, M.; MUNSEN, M. A. Ontologies in support of problem solving. In: STAAB, S.; STUDER, R. (Eds.). Handbook on ontologies. Berlin: Springer-Verlag, 2004. p. 321-342.

EVERMANN, J. M. Using design languages for conceptual modelling: the uml case. 2003. 394f. Tese (Scientific Philosopher Doctorate Thesis) - The Faculty of Graduate Studies, Management Information Systems, Westfüalische Wilhelms Universitü at Münster, Münster, 2003.

EVERMANN, J. Thinking Ontologically: Conceptual vs. Design Models in UML. Business Systems Analysis with Ontologies. Hershey: Idea Group, 2005. p. 82-104.

FETTKE, P.; LOOS, P. Ontological analysis of reference models. In: GREEN, P.; ROSEMANN, M. (Eds.). Business systems analysis with ontologies. Hershey: Idea Group, 2005. p. 56- 81.

FONSECA, F. The double role of ontologies in information science research. Journal of the American Society for Information Science and Technology, v. 58, p. 786-793, 2007.

FOX, M. S.; GRUNINGER, M. Enterprise modeling. 1998. Available from Internet: $\quad<$ http://www.eil.utoronto.ca/enterprise-modelling/papers/foxaimag98.pdf>. Access: 26 May 2009.

FRIGG, R. Models in science. 2006. Available from Internet: $<$ http://plato.stanford.edu/entries/models-science/>. Access: 18 July 2008.

GENESERETH, M. R.; NILSSON, L. Logical foundation of AI. San Francisco: Morgan Kaufman, 1987. 405p.

GREEN, P.; ROSEMANN, M. Ontological analysis of business systems analyis techniques: experiences and proposals for an enhanced 
methodology. In: GREEN, P.; ROSENMANN, M. (Eds.). Business systems analysis with ontologies. Hershey: Ideia Group, 2005. p.1-27.

GRUBER, T. What is an ontology? 1993. Available from Internet: $<$ http://www-ksl.stanford.edu/kst/what- is-an-ontology.html $>$. Access: 14 Sep. 2002.

GUARINO, N. Formal ontology and information systems. 1998. Available from Internet: <http://citeseer.ist.psu.edu/guarino98formal.html>. Access: 26 May 2009.

GUARINO, N.; GIARETTA, P. Ontologies and KBs, towards a terminological clarification. In: MARS, N. (Ed.). Towards a very large knowledge bases; knowledge building and knowledge sharing. [S.I.]: IOS Press, 1995. p. 2532.

GUARINO, N.; GUIZZARDI, G. In the defense of ontological foundations for conceptual modeling. Scandinavian Journal of Information Systems, v. 18, n.1, p. 115-126, 2006.

GUARINO, N.; WELTY, C. Evaluating ontological decisions with OntoClean. 2002. Available from Internet: <http://citeseer.ist.psu.edu/guarino02evaluating.html >. Access: 24 Mar. 2005.

GUIZZARDI, G.; WAGNER, G. Some applications of a unified foundational ontology in business modeling. In: GREEN, P.; ROSEMANN, M. (Eds.). Business systems analysis with ontologies. Hershey: Idea Group, 2005. p. 345-367.

HERRE, H. et al. General Formal Ontology (GFO): A foundational ontology integrating objects and processes. Part I: basic principles. Leipzig: Research Group Ontologies in Medicine (Onto-Med), University of Leipzig, 2006.

HOLTEN, R.; DREILING, A.; BECKER, J. Ontology-driven method engineering for information systems. 2004. Available from Internet: $<$ http://www.wi.uni-muenster.de/is/projekte/mw-kid/pdfs/Ontology-

Driven Method Engineering for Information Systems Development.pdf $>$. Access: 10 Mar. 2008.

JACOBSON, I. et al. Object-oriented software engineering: a use case driven approach. New York: Addison-Wesley, 1992. 552 p.

JARDINE, D. A. The ANSI/SPARC DBMS model. In: SHARE WORKING CONFERENCE ON DATABASE MANAGEMENT SYSTEMS, 2., 1976, Montreal. Proceedings... Amsterdam: North Holland, 1976. p.1-225.

KÜHNE, T. What is a model? 2005. Available from Internet: <http://drops.dagstuhl.de/opus/volltexte/2005/23/pdf/04101.KuehneTho mas1.Paper.pdf>. Access: 15 July 2008.

MASOLO, C. et al. WonderWeb Library of Foundational Ontologies. 2003. Available from Internet: 
<http://wonderweb.semanticweb.org/deliverables/documents/D17.pdf>.

Access: 11 July 2008.

MILTON, S.; KAZMIERCZAK, E. Enriching the ontological foundations of modeling in information systems. 2000. Available from Internet: <http://www.comp.mq.edu.au/isf99/MiltonKaz.htm>. Access: 25 May 2009.

MYLOPOULOS, J. Conceptual modeling and telos. 1992. Available from Internet:

$<$ http://www.cs.toronto.edu/ jm/2507S/Readings/CM+Telos.pdf $>$.

Access 15 Dec. 2007.

OBERLE, D. et al. An extensible ontology software environment. In: STAAB, S.; STUDER, R. (Eds.). Handbook on ontologies. Berlin: SpringerVerlag. 2004. p. 299-320.

OLIVEIRA, V. N. P. Uma investigação sobre a avaliação de modelagem conceitual baseada em ontologias: estudo de caso de modelos para sistemas de informação desenvolvidos na Universidade Federal de Minas Gerais. 2009. Available from Internet: $<$ http://www.bibliotecadigital.ufmg.br/dspace/handle/1843/ECID7V2JBW >. Access 15 Dec. 2010.

RUMBAUGH, J. et al. Object-oriented modeling and design. New York: Prentice Hall, 1991. 512 p.

SÁNCHEZ, D. M.; CAVERO, J. M., MARCOS, E. On models and ontologies. 2005. Available from Internet: $<$ http://kybele.escet.urjc.es/PHISE05/papers/sesionIV/SanchezCaveroMar cos.pdf> Access: 26 May 2009.

SAYÃO, L. F. Modelos teóricos em Ciência da Informação:abstração e método científico. Ciência da Informação, Brasília, v. 30, n. 1, p. 82-91, 2001.

SHANKS, G.; TANSLEY, E.; WEBER, R. Using ontology to validate conceptual models 2003. Available from Internet: <http://portal.acm.org/>. Access: 26 May 2009.

SMITH, B. The basic tools of formal ontology. 1998. Available from Internet: <http://portal.acm.org/>. Access: 1 Feb. 2004.

SMITH, B. Ontology and Informations Systems. 2003. Available from Internet: <http://www.ontology.buffalo.edu/ontology>. Access: 22 Jan. 2006.

SMITH, B.; WELTY, C. Ontology: towards a new synthesis. 2001. Available from Internet: <http://www.cs.vassar.edu/ weltyc/papers/foisintro.pdf>. Access: 21 May 2005.

SØERGUEL, D. Functions of a thesaurus, classification and ontological knowledge bases 1997. Available from Internet from: $<$ http://www.clis.umd.edu/faculty/soergel/soergelfctclass.pdf $>$. Access: 12 Dec. 2003. 
SoWA, J. F. Ontoloy. 2000. Available from Internet: <http://www.jfsowa.com/ontology/>. Access: 20 March 2006.

SYCARA, K.; PAOLUCCI, M. Ontologies in agent architectures. In: STAAB, S.; STUDER, R. (Eds.). Handbook on ontologies. Berlin: Springer-Verlag, 2004. p. 343-364.

VICKERY, B.C. Ontologies. Journal of Information Science, v. 23, n.4. p.227-286, Jan. 1997.

WAND, Y.; WEBER, R. An ontological evaluation of systems analysis and design methods. In: FALKENBERG, E; LINGREEN, P. (Eds.). Information system concepts: an in-depth analysis. North-Holland: Elsevier Science, 1989. p. 79-107.

- Mario Bunge's ontology as a formal foundation for information systems concepts. In: Studies on Mario Bunge's Treatise. Amsterdan: Radopi, 1990. p. 123-150.

WAND, Y.; STOREY, V. C.; WEBER, R. An Ontological Analysis of the Relationship Construct in Conceptual Modeling (1999). Available from Internet: <http://portal.acm.org/ >. Access: 26 May 2009.

WISSUSEK, B.; KLAUS, $\mathrm{H}$. On the foundations of the ontological foundation of conceptual modeling grammars: the construction of the Bunge-Wand-Weber Ontology (2005). Available from Internet: $<$ http://user.cs.tu-berlin.de/ wyssusek/Publications/WyssusekKlaus_2005_On-the-foundation-of-the-ontological-foundation.pdf $>$. Access: 26 May 2009.

YOUNG, J. W.; KENT, H. K. Abstract formulation of data processing problems. The Journal of Industrial Engineering, New York, v. 9, n. 6, p. 471-479, Nov./ Dec.1958. 